\title{
Roteiro prático para preceptoria em fisioterapia intensiva pediátrica de um hospital universitário ${ }^{1}$
}

\author{
Gisele da Silva Peixoto Zandona \\ Graduada em Fisioterapia UNIBAN-SP \\ Mestranda em Ciências da Saúde - UFGD. \\ Especialista em Fisioterapia Intensiva Pediátrica e Neonatal \\ Coordenadora RT da fisioterapia da UTIP da UFGD. \\ Preceptora da Fisioterapia materno-infantil da UFGD. \\ $\triangle$ giselefisioterapia@hotmail.com \\ Deisiane da Silva Mesquita. \\ Graduada em Enfermagem-FCAT. \\ Mestre em epidemiologia e Vigilância em Saude-Instituto Evandro Chaves/SVS/MS. \\ Esp. Saúde Pública-Uepa/Fiocruz. \\ Téc. do Centro de Informações e Estratégias em Vigilância em Saúde do Estado do Pará. \\ هeisi_mesquita@hotmail.com \\ Meyrilane Vicente de Laias Moreira \\ Graduada em Fisioterapia UNIGRAN - MS \\ $\triangle \underline{\text { meyrilane.moreira@ebserh.gov.br }}$ \\ Camila Fortes Corrêa \\ Graduada em Enfermagem - UFSM \\ Mestranda em Ciências da Saúde - UFGD. \\ Especialista em Enfermagem Intensiva Pediátrica e Neonatal- AVM \\ Especialista em Preceptoria em Saúde - UFRN \\ Preceptora da Enfermagem do Programa de Residência Multiprofissional da UFGD. \\ myllemila@yahoo.com.br \\ Shanna Machado de Sousa \\ Graduada em Enfermagem - UESPI \\ Especialista em Enfermagem em Terapia Intensiva \\ $\square$ shannamachado@hotmail.com
}

Recebido em 25 de agosto de 2021

Aceito em 23 de novembro de 2021

\begin{abstract}
Resumo:
É importante que o preceptor da unidade de terapia intensiva pediátrica tenha um planejamento estruturado para sequenciar os aprendizados com coerência dos temas e qualidade de conteúdo aos seus residentes, sendo esses os principais objetivos da preceptoria. Permitindo assim articular de forma prática e didática os diversos assuntos necessários para a formação concisa desse residente ao mesmo tempo, em que permite obter melhores parâmetros de avaliação. Este roteiro destina se também a desenvolver conhecimentos, habilidades, competências e atitudes necessárias à prática multiprofissional, possibilitando o desenvolvimento da capacidade crítica, reflexiva, participativa e transformadora, principalmente focada nas especificidades da área de concentração da residência. Este artigo trata-se de projeto de intervenção do tipo plano de preceptoria onde foi estruturado em formato de Roteiro Prático de Ensino e a avaliação quinzenal ou mensal foi seguindo a metodologia na pirâmide de Miller. 0 roteiro proposto foi resultado da interação com a rede de apoio com todos os atores da preceptoria; visando a melhoria na capacidade técnica assistencial desses residentes; e também a reversão das fraquezas antes imposta pela falta de argumentação teórico dos assuntos mais questionados durante o estágio e o planejamento para as atividades por meio do cronograma, possibilitou o cumprimento das
\end{abstract}

\footnotetext{
${ }^{1}$ Trabalho de conclusão de curso, Especialista em preceptoria em Saúde, Universidade Federal do Rio Grande do Norte,2020, Orientador Deisiane da Silva Mesquita.
}

Online | www.e-publicacoes.uerj.br/index.php/sustinere/

ISSN - 2359-0424 
temáticas, permitindo a organização dos processos de preceptoria, abordados e graduados por nível de dificuldades. Com utilização do método, foi possível a materialização de um conceito pedagógico e aumentou o impacto educacional das avaliações, bem como qualificou a formação dos residentes, permitindo assim um aprendizado direcionado.

Palavras-chave: Preceptoria, Serviço de Fisioterapia em Hospital, Estágio Clínico.

\title{
Preceptorship plan in pediatric intensive physical therapy at a university hospital
}

\begin{abstract}
:
It is important that the pediatric intensive care unit preceptor has a structured plan to sequence the learning with consistency of themes and content quality for its residents, which are the main goals of preceptorship. Thus allowing to articulate in a practical and didactic way the different subjects necessary for the concise formation of this resident at the same time, in which it allows to obtain better evaluation parameters. This script is also intended to develop knowledge, skills, competences and attitudes necessary for multidisciplinary practice, enabling the development of critical, reflective, participatory and transformative capacity, mainly focused on the specifics of the residency concentration area. This article is about an intervention project of the preceptorship plan type where it was structured in the format of Practical Teaching Guide and the biweekly or monthly evaluation followed the methodology in Miller's pyramid. The proposed script was the result of interaction with the support network with all the preceptorship actors; aiming to improve the technical assistance capacity of these residents; and also the reversal of the weaknesses previously imposed by the lack of theoretical argumentation of the most questioned issues during the internship and the planning for the activities through the schedule, enabled the fulfillment of the themes, allowing the organization of the preceptorship processes, addressed and graduated by level of difficulties. Using the method, it was possible to materialize a pedagogical concept and increased the educational impact of the assessments, as well as qualifying the training of residents, thus allowing for targeted learning.

Keywords: Preceptorship, Hospital Physiotherapy Service, Clinical Internship.
\end{abstract}

\section{Plan de preceptuación en fisioterapia intensiva pediátrica en un hospital universitario}

\begin{abstract}
Resumen:
Es importante que el preceptor de la unidad de cuidados intensivos pediátricos tenga un plan estructurado para secuenciar el aprendizaje con consistencia de temas y calidad de contenido para sus residentes, que son los principales objetivos de la preceptoría. Permitiendo así articular de manera práctica y didáctica los diferentes temas necesarios para la formación concisa de este residente al mismo tiempo, en lo que permite obtener mejores parámetros de evaluación. Este guión también tiene como objetivo desarrollar conocimientos, habilidades, competencias y actitudes necesarias para la práctica multidisciplinar, posibilitando el desarrollo de la capacidad crítica, reflexiva, participativa y transformadora, enfocada principalmente en las especificidades del área de concentración de la residencia. Este artículo es un proyecto de intervención tipo plan de preceptoría, que se estructuró en formato de Guía Práctica Docente y la evaluación quincenal o mensual siguió la metodología en la pirámide de Miller. El guión propuesto fue el resultado de la interacción de la red de apoyo con todos los actores de la tutela; con el objetivo de mejorar la capacidad de asistencia técnica de estos residentes; y también la reversión de las debilidades previamente impuestas por la falta de argumentación teórica de los temas más cuestionados durante la pasantía y la planificación de las actividades a través del cronograma, posibilitó el cumplimiento de los temas, permitiendo la organización de los procesos de preceptoría, abordados y graduado por nivel de dificultad. Con el método se logró materializar un concepto pedagógico y aumentar el impacto educativo de las evaluaciones, además de calificar la formación de los residentes, permitiendo así un aprendizaje focalizado.
\end{abstract}

Palabras clave: Preceptoría, Servicio de Fisioterapia Hospitalaria, Internado Clínico.

\section{INTRODUÇÃO}


A Unidade de Terapia Intensiva Pediátrica destina-se ao acolhimento de pacientes em estado grave com chances de sobrevida, que requerem monitoramento constante (24 horas) e cuidados complexos. A unidade acompanhada neste estudo atende pacientes entre 29 dias a 12 anos incompletos, sendo este limite definido conforme as rotinas hospitalares internas.

Este cenário permite a integração teoria-prática dos residentes de modo geral, conforme preconizado em estudos, bem como nas políticas públicas de saúde, sendo componente fundamental para promover mudanças na forma de ensinar e aprender, principalmente em promover cuidados no campo da saúde no Brasil (DILLON et al., 2003).

É perceptível que residentes e preceptores em parceria no serviço de saúde proporcionam o aprendizado significativo, ao mesmo tempo, em que estimulam a educação permanente para estes, a partir da troca de saberes, do incentivo à pesquisa, da reflexão sobre a prática e do estímulo ao trabalho colaborativo em equipe (MADRUGA et al., 2017).

O preceptor da unidade de terapia intensiva pediátrica exerce atribuições que são inerentes ao seu cargo, desenvolve também atividades de responsabilidades técnicas, coordenação de equipe, alinhamentos de condutas, avaliação e manejo de necessidade tecnológica em saúde. Neste cenário para ter êxito o fisioterapeuta preceptor necessita de um plano de preceptoria, o qual deve ser prático, rápido e eficaz.

Neste contexto, Pimentel (2019), reforça que o processo ensino-aprendizagem, precisa ser objetivo, com metas específicas e que isso irá favorecer a aquisição de competências nas atividades pratica do dia-dia do preceptor; relata ainda que a técnica: "Preceptor de um minuto" onde compõe um conjunto estruturado de cinco passos centrados no estudante; é satisfatório e contribui positivamente para o desenvolvimento das práticas; facilitando o raciocínio clínico e crítico dos estudantes e melhorando a condução e a oferta do feedback por parte dos preceptores.

Desta forma, o objetivo principal deste trabalho é propor um roteiro de estágio prático específico para os residentes da fisioterapia em UTI pediátrica. Este roteiro justifica-se devido à grande demanda de temas a serem abordados durante o estágio, de modo que este conclua seu estágio apto a conduzir os casos mais frequentes tanto em emergências pediátricas quanto nas Unidades de Terapia Intensiva Pediátricas (UTIPS). 
A proposta do roteiro foi referenciada na metodologia ativa, com aprendizagem baseada em problemas, onde está é potencialmente prática e dinâmica e contribui para que os preceptores em sua tarefa diária de ensino, possibilitem novos conhecimentos para os residentes. E também teve como referência a técnica Preceptoria de um minuto.

Diante da perspectiva dessas metodologias, esse artigo tem uma questão norteadora: a criação de um roteiro de estágio especifico para a fisioterapia intensiva pediátrica, onde abrange um público bem heterogêneo de causa para admissões, epidemiologias discrepantes entre os grupos e unidades, e abrangentes temas para serem abordados em todo contexto da terapia intensiva pediátrica (EINLOFT et al., 2002).

Com isso, vislumbra-se a consolidação de práticas educacionais pautadas na criticidade, reflexividade, contextualização e aprendizado significativo que impactem na atenção integral ao usuário e na qualidade dos serviços oferecidos.

\section{METODOLOGIA}

Trata se de um projeto de intervenção do tipo Plano de Preceptoria em formato de roteiro, criada seguindo a metodologia ativa do qual estimula a educação crítico-reflexiva com base em estímulo no processo ensino-aprendizagem, resultando em envolvimento por parte do educando na busca pelo conhecimento (MACEDO et al, 2018).

A avaliação dos temas abordados no roteiro, foi proposto baseando-se na pirâmide das competências, introduzida por Miller, que diretamente relaciona-se as meta-habilidades dos residentes. Já os instrumentos avaliativos foram: auto avaliação, trabalho em grupo, simulação de atendimentos com questões orais e testes com casos clínicos de forma dissertativa (MILLER, 1990).

Para Miller (1990), a avaliação é o modelo conceitual que ilustra as bases cognitivas ("saber" e "saber como fazer") da prática profissional ("fazer") e a necessidade da avaliação de habilidades e competências práticas ("mostrar como faz"). 
Os períodos avaliativos eram mensalmente e de forma aleatória usando um dos instrumentos acima citados, respeitando os conceitos que Miller (1990) traz que para exercer a profissão não é suficiente desenvolver apenas habilidades práticas, é essencial também a aplicação do conhecimento teórico, de modo a revelar sua importância em promover discernimento nas suas ações. 0 conhecimento é então a base da pirâmide.

A aplicação do roteiro foi realizada em um Hospital Universitário Federal, situado na cidade de Dourados/MS, tendo como público alvo os residentes de fisioterapia do Programa de Residência Materno Infantil.

Atualmente a linha Infantil do referido hospital é destinada à recuperação de pacientes pediátricos e neonatais que requeiram assistência hospitalar; dispondo de 10 leitos para a internação de crianças na faixa etária de 0 mês a 11 anos, 11 meses e 29 dias, com perfil clínico ou cirúrgico, provenientes do próprio hospital, da atenção básica municipal, dos pronto-atendimentos da rede macrorregional e demais hospitais do município e da região.

$\mathrm{Na}$ unidade recebemos em sua maioria, crianças com insuficiência respiratória aguda necessitando de suporte avançado tais como intubação orotraqueal, necessidade de ventilação mecânica, monitoramento de drenagem torácica, toracocentese, ventilação não invasiva, oxigenioterapia, cuidados intensivos gerais, e em menor número as admissões referentes a pósoperatórios imediatos de gastrectomia, apendicectomia, laparotomia, trauma crânio encefálico, tumores, desnutrição, desidratação, dentre outros.

As crianças admitidas na UTIPED do Hospital Universitário Federal de Dourados/MS com suspeitas de H1N1 e/ou COVID -19 são colocadas em isolamento respiratório e de contato, até resultado da coleta de aspirado traqueal ou Swab nasal. $\mathrm{Na}$ atualidade, em sua maioria os resultados destes vem diagnosticando outros vírus.

\section{RESULTADOS}

A elaboração do roteiro de preceptoria, foi planejado para o período de dois meses de estágio teórico e prático na UTI PED, o qual entendemos que este período seria o suficiente, porém não o ideal.

Para a inclusão dos temas no roteiro, foi realizado um levantamento dos assuntos mais frequentes questionados pelos residentes; do qual foi dividido por semanas e a complexidade graduada conforme as dificuldades relatadas pelos mesmos. 
Organização do roteiro:

\section{1.ํo mês:}

\section{Semana 1:}

1. Avaliação sobre os temas mais frequentes em terapia intensiva; com levantamento de questões, formulação de hipóteses de temas a serem abordados nas próximas semanas: ventilação mecânica invasiva (VMI) e ventilação mecânica não invasiva (VNI), gasometria arterial e demais exames laboratoriais e de imagem;

2. Realização de 2 orientações e sequenciamento de avaliação respiratória, motora e circulatória, seguida de prática assistencial à beira leito;

3. Promoção da prática na avaliação correlacionado com conduta fisioterápica;

4. Proposta de um tema a ser debatido com a equipe multidisciplinar para educação permanente.

\section{Semana 2:}

1. Realização de avaliação fisioterapêutica dos pacientes; envolvendo todos os contextos dos cuidados e reabilitação;

2. Discussão de um caso específico, baseando-se em evidências científicas atuais; seguindo a preceptoria de minuto;

3. Induzir o aluno a comprometer-se com o caso. "O que você faria nesse caso? ", "Qual a sua opinião? ";

4. Basear-se em conhecimentos científicos já adquiridos. Revisão teórica do tema com o aluno;

5. Referir-se a regras gerais; 
6. Reforçar o que foi feito corretamente.

\section{Semana 3:}

1. Busca de conteúdo prévio já aprendidos e estimular conduta baseada em evidências no público pediátrico (Manobras de higiene brônquica e manobras de reexpansão);

2. Integração do conteúdo à prática na realização de fisioterapia motora de acordo com avaliações: estudar posicionamento no leito e mobilização precoce no paciente pediátrico, atividade lúdicas, escalas de funcionalidade, dentre outros temas que envolvam a fisioterapia;

3. Registro em prontuário eletrônico as evoluções de forma completa e objetiva, e submissão desta avaliação ao preceptor diariamente com feedback avaliativo no mesmo momento;

4. Estimulo e avaliação diária do trabalho em equipe multiprofissional.

\section{Semana 4:}

1. Realização de fisioterapia respiratória e manobras de higiene brônquica (aprofundamento teórico nas manobras de higiene brônquica pediátrica e reexpansão pulmonar);

2. Realização de prática em ventilação mecânica (parâmetros, leitura gráfica, correção e identificação de assincronias, montagem de circuitos e ajustes finos);

3. Realização de prática de VNI entre os residentes e treino de ajustes e sincronias.

Decorrido 1 mês de estágio, deu-se a avaliação mensal: sendo esta elaborada com um caso clinico, especifico e composta pelos itens: avaliação respiratória e motora, interpretação de exames laboratoriais, bem como a conduta correlacionada com a clínica. 
Foi realizado também: avaliação da apresentação de uma atividade de educação permanente no setor; avaliação continuada do desenvolvimento acadêmico-assistencial dos residentes ao longo do mês e uma avaliação do desempenho no conhecimento prático adquirido, baseado nos problemas enfrentados durante o mês anterior. Sendo que a meotodologia usada para essas avaliaçoes foram através da Piramide de Miller, do qual preconiza se : "mostrar como se faz" realizado durante um atendimento prático, e conduzido com questões abertas de forma oral, e pontuado cada item da anamnese, conduta clínica e sugestões de melhorias do processo.

\section{2. ํôês:}

\section{Semana 1:}

1. Prática clínica com avaliação da fisioterapia respiratória, motora, aspiração de vias aéreas e posicionamento funcional no leito;

2. Proposta de 1 tema para o residente elaborar um Procedimento Operacional Padrão (POP);

3. Planejamento do conteúdo programático para o mês: atividades do eixo específico, assim como os objetivos de aprendizagem:

Dia 1: Discussão sobre Síndrome do Desconforto respiratório agudo pediátrico (PARDS) e como ventilar;

Dia 2: Ventilações protetoras, recrutamento alveolar e prevenção de LIVI (Lesão Induzida pela Ventilação Mecânica);

Dia 3: Tipos mais frequentes de insuficiência respiratória aguda em pediatria, suas causas e tratamento fisioterápicos: tipos de atelectasias e de pneumonias, associado a conduta multiprofissional.

Dia 4: Cardiopatias pediátricas;

Dia 5: Assincronias ventilatórias; 


\section{Semana 2:}

1. Treinamento de passagens de plantão e descrição no prontuário;

1. Ação de educação permanente;

2. Implementação de um POP.

\section{Semana:}

1. Motivação na participação do residente na tomada de decisões, envolvendo-o em todo o processo assistencial, e não somente na realização de técnicas;

2. Oportunizar e destacar procedimentos e técnicas específicas de cada clínica, além de orientar e supervisionar sua realização.

\section{4. . Semana:}

1. Estudos guiados e discutidos com artigos científicos com os temas: ventilação mecânica invasiva (modos e modalidades), bronquiolites, derrames pleurais e bronquiectasias.

\section{DISCUSSÕES}

Os desafios e dificuldades vivenciadas por preceptores brasileiros no processo educacional dos residentes, são citadas por pesquisadores, apresentando destaque para: o despreparo para atuar com metodologias ativas, infraestrutura inadequada e deficiente, dificuldades relacionadas à comunidade e à gestão do processo (LIMA, ROZENDO 2015; IZECKSON, et al 2017).

Tais achados corroboram com os encontrados neste estudo, principalmente no que refere aos vivenciados em nosso processo de preceptoria do qual necessitou sofrer alterações conforme as fragilidades e oportunidades na dinâmica do setor. 
Dentre as fragilidades que eventualmente ocorreram podemos citar: a sobrecarga de trabalho dos profissionais, com isso a falta de tempo hábil durante os plantões para discussões sobre os processos de trabalho junto aos residentes, porém mantendo sempre a intenção de que o processo ocorra de forma didática e correta; eventuais intercorrências no setor atrasando o cronograma do roteiro; a falta de colaboração ou aceitação dos colegas de trabalho para participar das discussões em equipe multiprofissional; tramites burocráticos para pesquisa clínica; ausência de preceptores das outras categorias profissionais e a falta de recursos humanos e/ou tecnológicos (LIMA, ROZENDO 2015;IZECKSON, et al 2017).

Após decorrido 1 mês de estágio prático a avaliação com a metodologia baseada em problematização, composta por um caso clínico específico e pelos seguintes itens: avaliação respiratória e motora, interpretação de exames laboratoriais, bem como a conduta correlacionada com a clínica. Sob essa metodologia Pissaia, (et al, 2018), justifica o uso desse método para avaliação prática clínica e traz que os estudantes compreendem a importância desse processo como uma forma de exercitar a própria aprendizagem e estes sentirem-se inseridos no processo integrando-se ao meio, na construção do saber.

A proposta de roteiro semiestruturado focada na relação preceptor-residente é descrita de forma prática, rápida e curta, de modo a alcançar os objetivos de cada categoria profissional (AGUIAR; 2017). O processo da preceptoria em formato de roteiro possibilitou estimular a reflexão e debate do tema com outras categorias, a prática do conteúdo teórico, a busca de temas que geram dificuldades generalizadas da equipe e estratégias para educação permanente.

As oportunidades geradas com o roteiro trouxeram incentivo a mais conteúdo técnico e teórico, às possibilidades de atividades multiprofissionais compartilhadas, ao estímulo a criatividade, o acompanhamento clinico, a trabalhadores qualificados no futuro, o incentivo a pesquisa e a autonomia no ensino.

\section{CONSIDERAÇÕES FINAIS}


Este ensaio demonstrou e contextualizou a importância da programação do conteúdo que foi abordado durante o estágio da residência, não apenas na prática tarefeira em relação ao desenvolvimento do serviço, mas como aprendizado, estimulando o raciocino clínico no tratamento do paciente como um ser holístico e interligando os vários olhares interdisciplinares.

Os benefícios alcançados com a elaboração do roteiro prático de ensino foram vários, dentre eles: o surgimento de rede de apoio com todos os atores da preceptoria, a melhoria da capacidade técnica assistencial, a reversão das fraquezas antes imposta pela falta de argumentação teórico de determinado tema, o tempo para as atividades no cronograma semanal, auxiliando no cumprimento de todos os objetivos, com discussões dos temas abordados e graduados por nível de dificuldades.

As limitações enfrentadas, que foram à falta de disponibilidade de tempo suficiente para execução do cronograma, porém, este foi revertido com a própria articulação no roteiro.

A utilização do método foi à materialização de um conceito pedagógico, para se poder aumentar o impacto educacional das avaliações. Há ainda, que se promover reflexões mais abrangentes sobre os conceitos subjacentes aos métodos, para se compreender como as informações obtidas possam de fato contribuir para a qualificação dos estudantes que pretendemos formar e colocar disponível no mercado de trabalho.

\section{REFERÊNCIAS}

AGUIAR, Adriana Cavalcanti. Preceptoria em Programas de Residência: ensino, pesquisa e gestão / Adriana Cavalcanti de Aguiar (Organizadora). Rio de Janeiro: CEPESC/IMS/UERJ, 2017. Disponível em: https://preceptores.icict.fiocruz.br/livro-preceptoria-em-programas-de-residencia.pdf. Acessado em 15 de novembro de 2020

BOTTI, Sérgio Henrique de Oliveira. O Papel do Preceptor na Formação de Médicos Residentes: um estudo de residências em especialidades clínicas de um hospital de ensino. Rio de Janeiro; 2009. Doutorado [Tese] Escola Nacional de Saúde Pública Sergio Arouca.). Disponível em: https://www.arca.fiocruz.br/bitstream/icict/2582/1/ENSP_Tese_Botti_Sergio_Henrique.pdf

DEMO, Pedro. Educação e qualidade. Campinas: Editora Papirus, 1994. (Coleção magistério: Formação e trabalho pedagógico). 
DILLON, Loretta Schoen; TOMAKA, Joseph W.; CHRISS, Claudia E; GUTIERREZ, Camelia P; HAIRSTON, Jennifer M. The effect of student clinical experiences on clinician productivity. J Allied Health, p. 261-5, 2003.Disponivel em:_https://pubmed.ncbi.nlm.nih.gov/14714600/. Acessado em 23 de março de 2021.

EINLOFT, Paulo Roberto; GARCIA, Pedro Celiny; PIVA, Jefferson Pedro; BRUNO, Francisco; KIPPER, Délio José; FIORI, Renato M. Perfil epidemiológico de dezesseis anos de uma unidade de terapia intensiva pediátrica. Revista de Saúde Pública, v. 36, n. 6, p. 728-733, dez. 2002. Disponível em : https://www.scielo.br/j/rsp/a/48kXd8Lbgyn7f4tjz3pPtmF/abstract/?lang=pt. Acessado em outubro de 2020.

IZECKSOHN, Mellina Marques Vieira; JUNIOR, Jorge Esteves Teixeira; STELET, Bruno Pereira; JANTSCH, Adelson Guaraci. Preceptoria em Medicina de Família e Comunidade: desafios e realizações em uma Atenção Primária à Saúde em construção. Ciência \& Saúde Coletiva [online]. 2017, v. 22, n. 3, pp. 737-746. ISSN 16784561.Disponivel em: https://www.scielo.br/j/csc/a/6TSMNrnJvwcSgjvwzGjRFmd/abstract/?lang=pt. Acessado em junho 2020.

LIMA, ROZENDO. Desafios e possibilidades no exercício da preceptoria do Pró-Pet Saúde. Interface 19 (suppl 1) • Ago 2015 • https://doi.org/10.1590/1807-57622014.0542 . Acessado em: 10 de março de 2021.

MADRUGA, Luciana Margarida de Santana; MARTINS, Fábio de Lima; BEZERRA, Samarony Caio Moreno; PASSOS, Anadélia Lopes de Souza; LIMA E COSTA, Murillo Frazão de; DIAS, Adriana Luna Pinto; LEITE, Karla Neves; COSTA, Thaísa Dias de Carvalho. O papel do fisioterapeuta na preceptoria de uma residência multiprofissional em saúde hospitalar. Cadernos de Educação, Saúde e Fisioterapia, v. 4, n. 8, 2017. Disponível em: http://revista.redeunida.org.br/ojs/index.php/cadernos-educacao-saude-fisioter/issue/view/32/showToc. Acessado em 5 de janeiro de 2020.

MILLER, G. E. The assessment of clinical skills/ competence/performance. Acad Med. 1990;65(9 suppl): S63S67.Disponivel em: https://journals.lww.com/academicmedicine/toc/1990/09000. Acessado em 30 de outubro de 2020.

MACEDO, Kelly Dandara da Silva; ACOSTA, Beatriz Suffer; SILVA, Ethel Bastos da; SOUZA, Neila Santini de; COLOMÉ, Carmem Lúcia; SILVA, Karla Kristiane Dames. Metodologias ativas de aprendizagem: caminhos possíveis para inovação no ensino em saúde. Esc. Anna Nery, Rio de Janeiro, v. 22, n. 3, 2018. Disponível em: https://www.scielo.br/j/ean/a/XkVvYBMtbgRMLxQvkQGqQ7z/?lang=en. Acessado em: 07 de abril de 2021.

PISSAIA, Luís Felipe; QUARTIERI, Marli Teresinha; AMADO, Nélia Maria Pontes; CARREIRA, Susana Paula Graça; REHFELDT, Márcia Jussara Hepp; COSTA, Arlete Eli Kuns da. Metodologia de problematização como processo avaliativo em um curso profissionalizante na área da saúde. Revista Sustinere, [S.1.], v. 5, n. 2, p. 279 - 295, jan. 2018. ISSN 2359-0424. Disponível em: https://www.epublicacoes.uerj.br/index.php/sustinere/article/view/30285. Acessado em: 07 de abril de 2021.

\section{(cc) BY}

Este trabalho está licenciado com uma Licença Creative Commons - Atribuição 4.0 Internacional. 